\title{
Etudes floristique et ethnobotanique des plantes médicinales de la ville de Kénitra (Maroc)
}

\author{
Souad Salhi, Mohamed Fadli, Lahcen Zidane \& Allal Douira (*)
}

Résumé: Salhi, S., Fadli, M., Zidane, L. \& Douira, A. Etudes floristique et ethnobotanique des plantes médicinales de la ville de Kénitra (Maroc). Lazaroa 31: 133-146 (2010).

Dans le but de connaître les plantes médicinales utilisées traditionnellement par la population de la ville de Kénitra (Nord ouest du Maroc), une étude floristique et ethnobotanique a été réalisée dans cette région.

L'étude de la flore médicinale a permis d'inventorier 62 espèces appartenant à 34 familles et 58 genres. De même, une série d'enquêtes ethnobotaniques réalisées à l'aide d'un questionnaire, a permis de collecter un certains nombres d'informations.

Les résultats de cette étude ont montré que le feuillage constitue la partie la plus utilisée. La majorité des remèdes est préparée sous forme de décoction. Sur l'ensemble des maladies traitées, les affections digestives représentent les maladies les plus citées.

Mots clés: Plantes médicinales, Ethnobotanique, Questionnaire, Médecine traditionnelle, Kénitra.

Abstract: Salhi, S., Fadli, M., Zidane, L. \& Douira, A. Floristic and ethnobotanical study of medicinal plants of Kénitra (Maroc). Lazaroa 31: 133-146 (2010).

The aim of this work is to determine the medicinal plants used traditionally by the population of Kenitra, a town located in northwest Morocco.

Research into medicinal flora allowed us to obtain 62 species belonging to 34 families and 58 types. Additional information was collected for ethnobotanical investigation by means of a questionnaire.

The results of this study showed that the most commonly used part of the plant is the foliage. Some remedies are prepared by decoction. Among the ailments treated, digestive problems were overall the most frequently mentioned.

Keywords: medicinal plants, ethnobotany, questionnaire, traditional medicine, Kenitra.

\section{INTRODUCTION}

Les plantes médicinales constituent un patrimoine précieux pour l'humanité et plus particulièrement pour la majorité des communautés démunies des pays en voie de développement qui en dépendent pour assurer leurs soins de santé primaires et leurs subsistances. Elles utilisent la plupart des espèces végétales, tant ligneuses qu'herbacées, comme médicaments. Une croyance bien répandue est que toute plante soigne.
Selon l'Organisation Mondiale de la Santé, plus de $80 \%$ des populations africaines ont recours à la médecine et à la pharmacopée traditionnelle pour faire face aux problèmes de santé. Le continent africain regorge de plantes médicinales très diversifiées. En effet, sur les 300.000 espèces végétales recensées sur la planète plus de 200.000 espèces vivent dans les pays tropicaux d'Afrique et ont des vertus médicinales (SoFOwORA, 1993).

Les plantes médicinales demeurent encore une source de soins médicaux dans les pays en voie

\footnotetext{
*Laboratoire de Botanique et de Protection des Plantes, Faculté des Sciences, B.P. 133, Université Ibn Tofail, Kénitra, Maroc.E-mail:douiraallal@hotmail.com; salhisouad@gmail.com
} 
de développement en l'absence d'un système médicinale moderne (TABUTI \& al., 2003).

Le Maroc par sa position biogéographique, offre une très grande diversité écologique et floristique. Il est l'un des pays méditerranéens qui ont une longue tradition médicale et un savoirfaire traditionnel à base de plantes médicinales (SCHERRER \& al., 2005). Par ailleurs, une forte tradition éthnomédicinale et encore vivante dans toutes les régions du Maroc et nul ne soupçonne la richesse du savoir éthnomédicinal accumulé durant des siècles et qui, de plus en plus risque de ne plus être transmis, en cas où aucune sauvegarde n'est entreprise.

La flore médicinale marocaine reste jusqu'à présent connue de manière simplement empirique. Devant cette parcelle de notre savoir, plusieurs volontés concourent, actuellement, à la préserver et à la mettre en valeur.

Par ailleurs, des enquêtes ethnobotaniques contribuent, de leurs parts, à rassembler et constituer une source d'information très précieuse, prête à être exploitée sur le plan scientifique.

Pour compléter des études partielles et fragmentaires qui ont été réalisées un peu partout au Maroc à savoir:

- Contribution à une étude ethnobotanique des plantes médicinales dans le Maroc oriental (KAHOUADJI, 1995)

- La pharmacopée marocaine, traditionnelle. Médecine arabe ancienne et savoir populaire (BELLAKHDAR, 1997)

- Etude ethnobotanique, phytochimique et pharmacologique des plantes aromatiques et médecinales du Tafilalt (EL RHAFFARI, 2002)

- Etude ethnobotanique de la flore médicinale dans la région de Rabat (Maroc occidental) (HSEINI \& KAHOUADJI, 2007)

Dans ce sens, une étude ethnobotanique se propose de mettre en évidence la place de la phytothérapie dans le système de soin traditionnel au niveau de la ville de Kénitra, située dans le Nord Ouest du Maroc.

Cette étude consiste à inventorier les plantes médicinales et à identifier les différentes manières d'utilisation et d'exploitation de ces dernières dans la médecine dite traditionnelle, familiale ou douce.
C'est dans cette optique que le présent travail s'inscrit et il a pour objectifs:

Etablir le catalogue des plantes médicinales utilisées par la population de la ville de Kénitra.

Recueillir l'ensemble des informations sur les applications thérapeutiques et traditionnelles locales.

Déterminer l'ensemble des maladies traitées.

\section{MATÉRIEL ET MÉTHODES}

\section{PRÉSENTATION DE LA RÉGION D’ÉTUDE}

La ville de Kénitra appartient au domaine de la plaine du Gharb, elle est située dans le coin Sud-ouest de ce dernier, à une distance de $12 \mathrm{Km}$ de l'océan Atlantique vers l'Ouest, $40 \mathrm{~km}$ de Rabat vers le Sud, $247 \mathrm{~km}$ de Tanger vers le Nord et $130 \mathrm{~km}$ de Meknès vers l'Est (Figure 1).

Sur le plan géologique, la plaine du Gharb correspond à un fossé d'effondrement situé entre deux grands ensembles structuraux, qui sont le domaine Mésétien primaire au Sud et le domaine de la chaîne rifaine au Nord et à l'Est. Cette plaine est marquée principalement par une subsidence continue depuis le Miocène (MiCHARD, 1976).

La couverture de la surface de la plaine du Gharb, proprement dite, est formée dans sa totalité par les dépôts du Gharbien dont les terrains anciens forment les zones intermédiaires et les zones basses. Le Gharbien récent constitue les zones de levées alluviales des bords des principaux cours d'eau (Sebou et Beht, Allouza, 2002).

Pour le réseau hydrographique, la plaine du Gharb s'inscrit dans le bas bassin du Sebou. Elle est traversée par le cours du bas Sebou et reçoit ses principaux affluents dont le Beht sur la rive droite. Ces cours d'eau génèrent des apports annuels de l'ordre de $6.10^{6} \mathrm{~m}^{3}$, soit environ $27 \%$ du potentiel hydraulique national mobilisable (HAIDA, 2000). Deux nappes superposées se distinguent dans la plaine du Gharb: une nappe phréatique, à faible potentialité et de mauvaise qualité et une nappe profonde en charge, assez productive et de bonne qualité (El MANSOURI, 1999). 


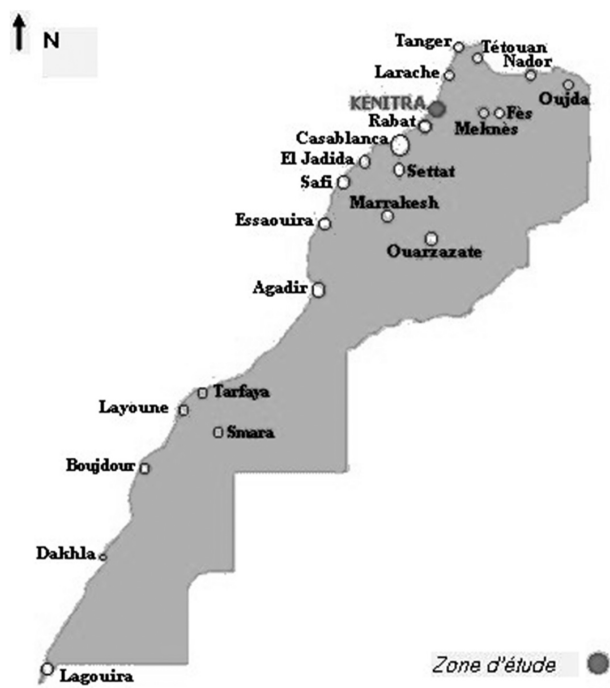

Figure 1.- Situation géographique de la zone d'étude.

Au niveau de la zone d'étude, c'est la nappe de Mamora qui constitue la principale ressource souterraine.

Le climat de la ville de Kénitra est de type méditerranéen, sur la base du quotient pluviométrique d'Emberger, elle appartient à l'étage bioclimatique subhumide à hiver tempéré.

\section{MÉTHOdOLOGIE}

L'étude ethnobotanique des plantes médicinales de la ville de Kénitra est effectuée d'une part selon un plan d'échantillonnage et d'autre part à l'aide d'un questionnaire pour bien cerner le problème et d'avoir une vue d'ensemble sur les utilisations traditionnelles locales et sur la diversité floristique de la pharmacopée de cette ville.

\section{Echantillonnage}

La ville de Kénitra est composée de douze annexes administratives regroupées en quatre districts urbains selon le découpage administratif en 2006 (Figure 2).

Notre échantillon d'étude est composé de $\mathrm{N}=$ 200 personnes, qui répondent aux principales caractéristiques de la population de la ville de Kénitra, afin d'avoir une meilleure représentativité de cette dernière.

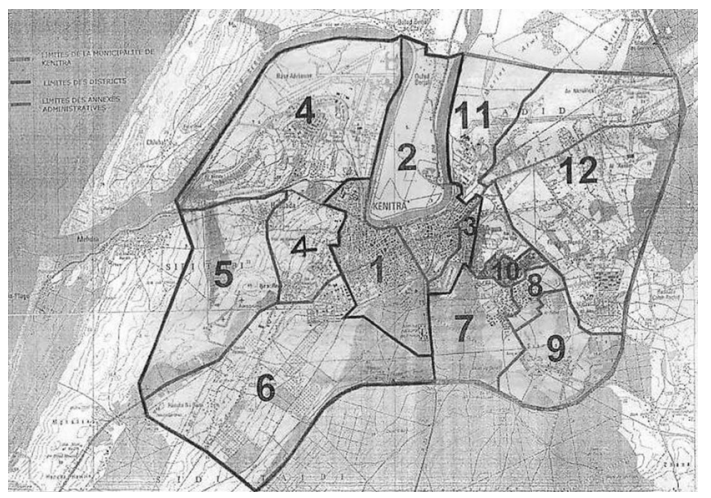

\section{Legende:}

District Maâmora (1,2 et 3)

District Oulad Oujeh (4, 5 et 6$)$

District Saknia (7,8 et 9)

District El Assam (10, 11 et 12)

Figure 2.- Découpage administrative de la ville de Kénitra.

Dans ce travail, l'échantillon est élaboré grâce à un mode d'échantillonnage probabiliste (aléatoire), il est divisé en groupes ou strates, donc on a quatre strates qui correspondent aux nombres des Districts urbains de la ville de Kénitra.

En procédant par un échantillonnage aléatoire stratifié, des échantillons de 50 personnes sont ensuite formés pour chacune des quatre strates et ils sont mis ensemble pour constituer l'échantillon global (200 personnes).

La proportion du nombre de personnes de chaque échantillon est la même (50 personnes) dans chaque strate, on parle donc d'un échantillonnage stratifié proportionnel (Tableau I).

Tableau 1

Répartition des enquêtés par chaque strate

\begin{tabular}{ccc}
\hline & Noms des Strates & Nombre d'enquêtés/Strate \\
\hline Strate 1 & Maâmora & 50 \\
Strate 2 & Oulad Oujeh & 50 \\
Strate 3 & Saknia & 50 \\
Strate 4 & El Assam & 50 \\
Echantillon & 200 \\
\hline
\end{tabular}




\section{Outil de recherche utilisé: le questionnaire}

L'étude ethnobotanique est effectuée suite à une série d'enquêtes réalisées à l'aide d'un questionnaire préétablie comportant des questions précises sur l'informateur, l'identité vernaculaire de la drogue végétale ainsi que la partie utilisée, les modes de préparation et les usages thérapeutiques et traditionnelles (Annexe 1).

L'enquête a permis d'interroger 200 personnes âgées de 18 à plus que 50 ans, répartie en 125 femmes contre 75 hommes et à des niveaux intellectuels différents, qui nous ont informé sur les applications thérapeutiques et traditionnelles locales de la population de la ville de Kénitra.

\section{RÉSULTATS ET DISCUSSION}

Selon ces enquêtes, nous avons rapporté 62 espèces végétales reparties en 58 genres et 34 familles, l'ensemble des ces espèces seront représentées dans le catalogue (Annexe 2).

Pour l'utilisation des plantes médicinales, les résultats obtenus montrent que ce sont les femmes qui les utilisent plus, ce résultat est conforme aux résultats obtenus ailleurs par divers auteurs (Kahouadj, 1995; HMAmouchi, 2001). Donc ce sont les femmes qui utilisent beaucoup plus les plantes médicinales que les hommes.

\section{ANALYSE DU CATALOGUE DES PLANTES MÉDICINALES}

\section{Aspect floristique}

L'analyse floristique du catalogue réalisé a permis de différencier 55 espèces appartenant à 32 familles botaniques.

En outre, l'identification botanique a montré que parmi les 34 familles recensées, celles les plus représentées sont les Lamiaceae (14 espèces soit 22,58\%), les Asteraceae (4 espèces soit $6,45 \%$ ), les Apiaceae (3 espèces soit $4,84 \%$ ) et les Caryophyllaceae ( 3 espèces soit 4,84\%). Les autres familles restantes ne comptent qu'une ou deux espèces (38 espèces soit $61,29 \%$ )

\section{Aspect ethnobotanique et pharmacologique}

Partie utilisée

Les parties végétales utilisées sont classées par ordre d'importance décroissante : les feuilles $(35 \%)$, les racines $(17,5 \%)$, la graine $(12,50 \%)$, la tige $(11,25 \%)$, l'inflorescence $(8,75 \%)$, la plante entière $(7,5 \%)$, et les fruits $(5 \%)$. Le reste des parties utilisées est représenté par un taux de $2,5 \%$ (Figure 3).

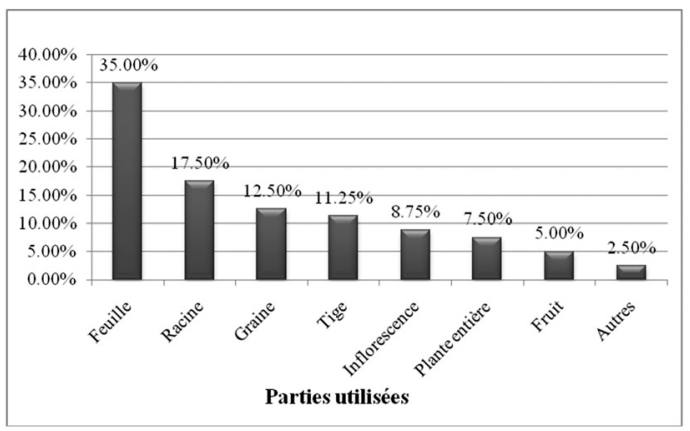

Figure 3.- Représentation des pourcentages des parties utilisées.

La fréquence d'utilisation élevée de feuilles peut être expliquée par l'aisance et la rapidité de la récolte (BITSINDOU, 1986) mais aussi par le fait qu'elles sont le siège de la photosynthèse et parfois du stockage des métabolites secondaires responsables des propriétés biologiques de la plante (BigendaKo-Polygenis \& LeJoly, 1990).

Mode de préparation

Afin de faciliter l'administration de la drogue, plusieurs modes de préparation sont employés à savoir la décoction, l'infusion, la poudre, la fumigation, le cataplasme, la macération et le badigeonnage. Les utilisateurs cherchent toujours la méthode la plus simple pour préparer les phytomédicaments.

La décoction aqueuse $(37,6 \%)$, l'infusion $(24,7 \%)$ et la poudre $(25,9 \%)$ sont les modes de préparation les plus utilisés (Figure 4). La décoction permet de recueillir le plus de principes actifs et atténue ou annule l'effet toxique de certaines recettes. 


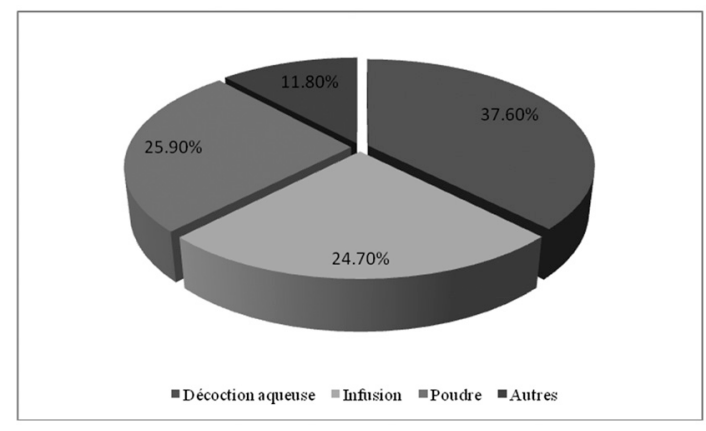

Figure 4.- Répartition des modes de préparation.

\section{Maladies et médecine traditionnelle}

Ce travail, qui contribue à une meilleure connaissance des soins traditionnels pratiqués dans la ville de Kénitra, nous a permis de répertorier un certain nombre de maladies chroniques traitées par les plantes médicinales. Les résultats obtenus montrent que la plupart des plantes interviennent dans le traitement des affections digestives $(26,15 \%)$, les affections dermatologiques (10\%), les affections obstétriques et gynécologiques $(17,70 \%)$ et les affections respiratoires $(16,15 \%$, Figure 5). Ce résul-

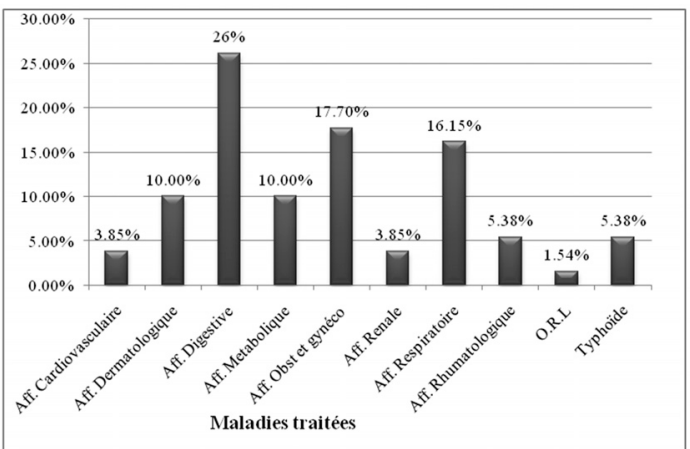

Figure 5.- Pourcentage des espèces répertoriées selon le groupe des maladies traitées. tat est conforme à celui obtenu par HMAMOUCHI \& Agoumi (1993).

\section{CONCLUSION}

La phytothérapie traditionnelle, était et reste actuellement sollicitée par la population ayant confiance aux usages populaires et n'ayant pas les moyens de supporter les conséquences de la médecine moderne. Ceci sans omettre l'important retour actuel vers la médecine douce.

Ainsi, le présent travail a été mené dans le but de réaliser un inventaire le plus complet possible des plantes médicinales utilisées dans la ville de Kénitra et de réunir les informations concernant les usages thérapeutiques pratiqués dans cette ville. La série d'enquêtes ethnobotaniques a permis de révéler une multitude de résultats.

Les résultats obtenus montrent que parmi les 34 familles recensées, la famille des Lamiaceae est la plus représentée entre elles par un effectif de14 espèces (soit 22,58\%). De point de vue ethnobotanique et pharmacologique, le feuillage constitue la partie la plus utilisée, la décoction est la forme galénique la plus pratiquée. De même, sur l'ensemble des maladies traitées, les affections digestives représentent les maladies les plus citées.

En outre, cette étude a permis d'apprécier et de connaître les pratiques traditionnelles utilisées par la population de la ville de Kénitra. La richesse de ce savoir-faire apparaît à travers les résultats obtenus mais il est important, d'une part d'étendre ce genre d'investigations à d'autres régions du pays afin de sauvegarder ce patrimoine culturel précieux par une monographie la plus complète possible; et d'autre part valider expérimentalement les remèdes recensés par des protocoles scientifiques rigoureux. 


\section{RÉFÉRENCES}

Allouza, M. -2002 - Evolution morphologique et sédimentologique de la frange littorale de la région de Kénitra: Bilan sédimentaire - Mém. Dipl.Etud. Sup. App. (ined.). Univ. Ibn Tofail. Fac. Sci., Kénitra, Maroc, 109 pp.

Bellakhdar, J. - 1997 - La pharmacopée marocaine, traditionnelle. Médecine arabe ancienne et savoir populaire - Ed. le Fennec. Casablanca, Maroc. 129-533.

Bigendako-Polygenis, M.J. \& Lejoly, J. - 1990 - La pharmacopée traditionnelle au Burundi. Pesticides et médicaments en santé animale. Pres. Univ. Namur. Pp. 425-442.

Bitsindou, M. - 1986- Enquête sur la phytothérapie traditionnelle à Kindamba et Odzala (Congo) et analyse de convergence d'usage des plantes médicinale en Afrique centrale - Mem. Doc (inéd.). Univ. Libre de Bruxelles. 482 pp.

Bruneton, J. - 1996- Plantes toxiques: végétaux dangereux pour l'homme et les animaux - Ed. Technique et Documentation, Paris, France.

Charnot, A. - 1945 - Toxicologie au Maroc - Mém. Soc. Sci. Nat. Ed. Siège de l'I.S., Rabat, Maroc. 717 pp.

Delaveau, P. - 1974 - Plantes et poisons végétaux - Horizons de France, Paris.

El Mansouri, B. - 1999- Développement d'outils et de concepts pour la gestion des eaux souterraines. Application à l'aquifère côtier du Gharb. Mém. Doc. Etat. Univ. Ibn Tofail, Fac. Sciences, Kénitra, Maroc. 145.

El Rhaffari, L. - 2002 - Etude ethnobotanique, phytochimique et pharmacologique des plantes aromatiques et médicinales du Tafilalt. Ressources végétales des oasis du sud-est du Maroc. Etat des lieux, valorisation gestion et préservation - Mem. Doc. Etat. (ined.). Univ. My Ismâil, Fac. Sci. Meknès, Maroc.
Haida, S. - 2000 - Transport de matières et bilan de l'érosion mécanique et de l'altération chimique dans un bassin versant d'une zone semi-aride : le Sebou. Impacts des variations climatiques et des activités humaines Mem. Doc. Etat. (ined.). Fac. Sci. Univ. Ibn Tofail. Kénitra, Maroc. 267.

Hmamouchi, M. - 2001 - Les plantes médicinales et aromatiques marocaines, $2^{\text {ème }}$ éd. 389 pp.

Hmamouchi, M. \& Agoumi, A. - 1993- Place des plantes médicinales dans le système de santé au Maroc - Prem. Congr. Int. Plant. Méd. phytothérap. $1^{\text {st }}$ Congr. Intercont., Tunis.

Hseini, S. \& Kahouadji, A. -2007- Etude ethnobotanique de la flore médicinale dans la région de Rabat (Maroc occidental) - Lazaroa 28: 79-93.

Kahouadji, M.S.-1995- Contribution à une étude ethnobotanique des plantes médicinales dans le Maroc orientale - Mem. Doc. $3^{\text {ème }}$ cycl. (ined.). Fac. Sci., Univ. Mohamed I, Oujda, Maroc. 206 pp.

Michard, A. - 1976 - Eléments de géologie marocaine Not. \& Mém. Serv. Géol. Maroc 252: 1-422.

Scherrer, A.M., Motti, R. et Weckerle, C. S. -2005- Traditional plant use in the areas of Monte Vesole and Ascea, Cilento National Park (Campania, Southern Italy) - J. Ethnopharmacol. 97: 129-143.

Sofowora, A. - 1993- Medicinal plants and traditional medicine in Africa, 2 - Spectrum Books Limited, Ibadan, Nigeria, 289.

Tabuti, J.R.S., Lye, K.A. \& Dhillion, S.S. -2003 - Traditional herbal drugs of Bulamogi, Uganda: plants, use and administration - J. Ethnopharmacol. 88: 19-44. 
Informateur:

\section{ANNEXE 1 : FICHE TECHNIQUE \\ Questionnaire \\ Plantes médicinales et phytothérapie}

- Age:

- Situation familiale:

- Sexe:

$\begin{array}{lll}\text { Célibataire ! } & \text { Marié ! } \\ \text { Masculin ! } & \text { Féminin ! } \\ \text { Néant } & \text { ! } & \text { Primaire ! }\end{array}$

Secondaire ! Universitaire !

Matériel végétal:

- Nom vernaculaire:

- Nom scientifique:

- Usage de la plante:

- Plante seule ! Association possible (de plantes) !

- Partie utilisées: Tige! Fleurs ! Fruits ! Graine ! Écorce ! Rhizome !

Bulbe ! Feuilles ! Plante entière !

Autres combinaisons ! :

- Forme d'emploi: Tisane ! Poudre ! Huiles essentielles !

Huiles grasses ! Extrait (teinture, solution, gélule)!

- Mode de préparation: Infusion! Décoction! Cataplasme ! Cru !

\section{Cuit ! Autres ! :}

- Dose utilisée:

- Pincée！ Poignée ! Cuillerée !

- Dose précise : Quantité en g/ verre:

Quantité en g/ litre:

Autres:

- Mode d'administration: Oral ! Massage ! Rinçage ! Badigeonnage !

Autres !:

- Posologie: nombre de prise par jour.

- Pour les enfants: 1fois/jour ! 2fois/jour ! 3fois/jour ! Autres ! :

- Pour les personnes âgées: 1fois/jour ! 2fois/jour ! 3fois/jour ! Autres ! :

- Pour les Adultes: 1fois/jour ! 2fois/jour ! 3fois/jour ! Autres ! :

- Durée d'utilisation (durée de traitement) :

Un jour ! Une semaine ! Un mois ! Jusqu’à la guérison ! .

\section{Utilisation:}

- Type de maladie:

- Affections dermatologiques

- Affections respiratoires

- Affections cardio-vasculaires

- Affections génito-urinaires

- Affections ostéo-articulaires

- Affections métaboliques

- Affections du tube digestif

- Affections des glandes annexes du tube digestif

- Affections neurologiques

- Diagnostic par:

Lui-même ! Le médecin ! L'herboriste ! Autres !:

- Résultats: Guérison! Amélioration ! Inefficace !

- Effets secondaires:

- Toxicité:

- Précaution d'emploi: 
ANNEXE 2:

\section{CATALOGUE DES PLANTES MEDICINALES}

\section{Anacardiaceae}

Pistacia lentiscus L. (Lentisque; Dro)

- Un décocté de feuilles sert au traitement des brûlures gastriques.

- Un décocté de racines est conseillé en cas d'asthme.

\section{Apiaceae}

Ammi visnaga Lam. (Khella; Bachnikha)

Un infusé de 15 ombelles dans un litre d'eau en cas de la constipation, un verre par jour jusqu'à avoir des selles normales.

Une ombelle dans un 1/4 litre d'eau froide (après 12 heures) à prendre à jeun pour traiter l'hypercholestérolémie.

Un décocté de plante entière est utilisé contre lithiases rénales.

Une fumigation d'ombelles est indiquée en cas de la fièvre typhoïde.

Eryngium tricuspidatum L. (Panicaut tricuspide; Mghizla)

Un décocté de racines est utilisé pour calmer les douleurs d'accouchement.

Foeniculum vulgare P. Mill. (Fenouil; Nafaâ, Bessbass)

Une décoction de graines est utilisée dans le traitement des douleurs gastro-intestinales et en cas d'asthme.

Une infusion de graines est utilisée en cas de ballonnement intestinal.

Les racines en poudre, mélangées au miel sont utilisées en cas de douleurs du côlon.

Toxicité. L'anéthol, principe de l'huile essentielle de fenouil, mal dosé, a provoqué, quelques accidents chez des personnes déjà mal en point du fait de l'éthylisme (BELlaKHDAR, 1997).

\section{Aristolochiaceae}

Aristolochia longa L. (Aristoloche; Berez'tem)
Le rhizome en poudre mélangé avec du miel ou du "Smen" est utilisé en cas de cancers (une cuillère par jour pendant 2 à 3 mois). Il est aussi indiqué en cas de diabète et d'asthme.

Une décoction est utilisée comme stimulante de l'appétit.

Toxicité. La plante, utilisée sur une certaine durée, provoque des lésions rénales irréversibles avec hématuries ainsi que des paralysies des membres (CHARNOT, 1945).

\section{Asparagaceae}

\section{Asparagus officinalis L. (Asperge; Seckoum)}

Une décoction à base de 3 à 4 rhizomes d'asperge et de chiendent (Agropyrum repens $\mathrm{P}$. Beauv.) pour un litre d'eau, faire bouillir $30 \mathrm{~min}$, filtrer. Une tasse par jour le matin pour traiter la stérilité féminine.

En cas de règles douloureuses, une infusion de rhizomes est conseillée.

Toxicité: La consommation des baies peut provoquer des troubles gastro-intestinaux de gravité variable (DelaVEau, 1974).

\section{Asphodelaceae}

Asphodelus microcarpus Viv. (Asphodèle; Berwag)

Le tubercule est creusé et rempli par quelques gouttes d'huile d'olive, après le chauffage du tubercule, l'huile est utilisée comme gouttes auriculaires en cas d'otite.

\section{Asteraceae}

Artemisia herba alba Asso (Armoise blanche; Chih)

En usage externe, la poudre d'armoise blanche est employée en cataplasme comme cicatrisante des brûlures et des plaies.

La décoction de parties aériennes, est efficace en cas de problèmes digestifs (ballonnement intestinal et parasitoses intestinales). 
Une décoction est utilisée en cas de règles douloureuses.

Toxicité: De fortes doses de la plante ont provoqué des cas d'intoxications, en particulier chez le nourrisson, l'enfant et la femme enceinte (BELLAKHDAR, 1997).

Atractylis gummifera L. (Chardon à glu; Addad)

A faible dose, la décoction de racines arrête les hémorragies et facilite l'accouchement.

A forte dose, il provoque l'avortement.

En usage externe, la poudre de racines est utilisée dans le traitement de la gale, des boutons d'acné, des chancres syphilitiques et des furoncles.

Le chardon à glu en poudre, associé au henné (Lawsonia inermis L.), est utilisé comme assouplissant des cheveux et traitement antipelliculaire.

Toxicité: Il est important de noter que cette plante est très toxique.

Inula viscosa Ait. (Inule visqueuse; Terrahla, Bagraman)

Une décoction de racines et de feuilles, à usage externe, soulage les douleurs rhumatismales.

Les feuilles en poudre ont un effet cicatrisant des plaies.

Matricaria chamomilla L. (Camomille; Babounje)

Une décoction est utilisée en cas de troubles digestifs.

Les capitules entre dans la préparation des teintures pour cheveux.

\section{Brassicaceae}

Lepidium sativum L. (Cressonnette; Hobb rchad)

Les graines sont indiquées dans les cas suivants: asthme, tuberculose, refroidissements, impuissance sexuelle, stérilité et syphilis en stade précoce.

Une bouillie de lait contenant de Cressonnette est utilisée comme fortifiant après l'accouchement.
Une petite cuillère de Cressonnette dans un verre de lait chaud, après 12 heures, à prendre en cas de bronchite.

Toxicité: Par leur huile essentielle, les graines, prises en grande quantité, peuvent provoquer des irritations des muqueuses (BELLAKHDAR, 1997).

\section{Capparidaceae}

Capparis spinosa L. (Câpre; Kabbar)

Une préparation à base de câpre, d'origan (Origanum compactum Benth.) et de graines de radis (Raphanus sativus L.) (Le tout en poudre) ajoutés au miel est utilisée en cas de stérilité féminine.

\section{Caryophyllaceae}

Corrigiola telephiifolia Pourret (Sarghine; Sarghina)

Les racines bien lavées puis réduites en poudre sont utilisées contre le rhumatisme.

Une cuillère à café de la poudre de racines dans un verre d'eau à apprendre en cas de la diarrhée.

Une décoction de sarghine est conseillée en cas de douleurs gastro-intestinales.

Herniaria hirsuta L. (Sabline rouge; Harras lahjar)

Un décocté de cette plante est conseillé en cas de lithiases rénales.

En usage externe, la sabline rouge en poudre mélangée avec l'huile d'olive est appliqué comme traitement antitaches (éviter l'exposition au soleil).

Saponaria officinalis L. (Saponaire; Tighcht)

En décoction, la saponaire est employée pour le traitement des troubles hépatiques et biliaires et en cas d'infections urinaires.

C'est un bon remède des affections pulmonaires (bronchite, toux).

\section{Chenopodiaceae}

Chenopodium ambrosioides L. (Anserine; Mkhinza) 
Une décoction de tiges et de feuilles est utilisée dans le traitement des douleurs gastro-intestinales, du ballonnement intestinal et des parasitoses intestinales (ascaridiose).

Le jus de feuilles fraîches mélangé avec le jus d'orange est utilisé en cas de fièvre, le cataplasme en feuilles joue le même rôle.

\section{Cucurbitaceae}

Citrullus colocynthis (L.) Schrad. (Coloqhinte; Lhdej)

Les graines séchées sont utilisées dans le traitement du diabète (deux à trois graines par jour le matin).

La pulpe des fruits écrasés s'applique localement sur les zones douloureuses en cas de rhumatisme.

Ecballium elaterium (L.) A. Rich. (Concombre d'âne; Faggous el-hemar)

Le fruit est écrasé dans le nez en cas d'ictère secondaire à une hépatite.

\section{Cupressaceae}

Tetraclinis articulata (Vahl.) Masters (Thuya; ârâr).

La décoction de poudre des rameaux mélangée à la poudre de l'écorce de lentisque (Pistacia lentiscus L.) est utilisée en bain contre la fièvre infantile.

Les feuilles en poudre associées au henné (Lawsonia inermis L.) sont appliquées en cataplasme, sur le cuir chevelu comme adoucissante et comme traitement anti-chute.

Le cataplasme de feuilles est utilisé en cas de migraines.

Une décoction de feuilles est indiquée dans le traitement des douleurs gastro-intestinales.

\section{Euphorbiaceae}

Mercurialis annиa L. (Mercuriale annuelle; Harrayga malsa).

Une infusion de feuilles est conseillée en cas de douleurs dorsales et gastro-intestinales.

Une fumigation de feuilles est conseillée en cas de douleurs uro-génitales.

\section{Fabaceae}

Glycyrrhiza glabra L. (Réglisse; ârq sûs)

Une préparation à base des racines de réglisse, des graines de fenouil (Foeniculum vulgare $\mathrm{P}$. Mill.), de lin (Linum usitatissimum L.) et de nigelle (Nigella sativa L.), sous forme de poudre, est mélangée avec du miel pour traiter l'asthme.

Une décoction de racines est utilisée en cas d'ulcère gastrique et d'infections urinaires.

Toxicité: L'abus de consommation peut entrainer une hypokaliémie sévère avec une hypertension artérielle et parfois fibrillation ventriculaire (BELlAKHDAR, 1997).

Trigonella foenum-graecum L. (Fenugrec; Halba)

Cette précieuse plante, offre de nombreux remèdes en cas de bronchite, diarrhée, diabète, kyste d'ovaire, gastralgies, maladies cardio-vasculaires.

Il est conseillé en cas d'allaitement.

\section{Gentianaceae}

Centaurium spicatum (L.) Fritsch (Petite centaurée; Kosset el haya)

Une décoction est utilisée en cas de douleurs gastriques.

\section{Hyacinthaceae}

Urginea maritima (L.) Baker (Urginée maritime; Bassila)

Une décoction de bulbe est utilisée en massage pour soulager les douleurs rhumatismales.

\section{Lamiaceae}

Ajuga iva (L.) Schreber (Ivette, Bugle; Chendgûra)

Le décocté et l'infusé ont prouvé leur efficacité dans le traitement de l'hypertension artérielle, le diabète, le cancer et l'asthme.

La poudre de feuilles sous forme d'infusion est utilisée en cas de traitement des douleurs gastriques.

L'infusion est conseillée comme un traitement de longue durée (plusieurs semaines) en cas de stérilité féminine. 


\section{Calamintha officinalis L. (Calament; Manta)}

Une décoction est recommandée en cas de grippe, de toux et de douleurs gastriques (deux tasses par jour).

\section{Lavandula dentata L. (Lavande; Lakhzama)}

Une décoction de sommités fleuries est conseillée en cas d'infection uro-génitale, de refroidissement, de règles douloureuses, de toux, de bronchite, rhumatisme et en cas d'une digestion difficile.

Un cataplasme de poudre de sommités fleuries est utilisé en cas de migraines.

Une fumigation est indiquée en cas d'infection génitale.

Lavandula stoechas L. (Lavande stoechade; Halhal)

Une décoction de lavande stoechade soulage les gastralgies et calme la toux.

Marrubium vulgare L. (Marrube blanc; Marriout)

La décoction de marrube dans le lait ou la soupe est utilisée pour soulager les affections respiratoires: toux, rhume, grippe et bronchite.

La décoction de marrube soulage les gastralgies.

Les feuilles de cette plante sont utilisées sous forme de cataplasme sur le front en cas de migraines et fièvre.

Une fumigation a un intérêt thérapeutique en cas de fièvre typhoïde.

\section{Mentha pulegium L. (Menthe pouliot; Fliyou)}

La tige feuillée en infusion ou en décoction dans du lait ou du thé est utilisée en cas de refroidissement et d'affections respiratoires (grippe, rhume et bronchite).

L'infusion de feuilles et branches fleuries est employée en cas de règles douloureuses, de douleurs abdominales et pour soulager les douleurs rhumatismales.

Les feuilles séchées roulées en cigarettes sont fumées pour soulager l'asthme.

Les feuilles et branches fleuries sont utilisées, en fumigation, en cas d'infection uro-génitale.

En cas d'allaitement, une décoction au lait est conseillée.
Mentha rotundifolia L. (Menthe ronde; Marsita, Timijja)

La région du Gharb est connue par les pains à la menthe ronde.

Les feuilles sont utilisées, en infusion, associées à l'armoise blanche (Artemisia herba alba Asso) et à l'origan (Origanum compactum Benth) contre les douleurs gastriques et en cas de fièvre et de la toux.

Une décoction de feuilles est utilisée pour l'hygiène intime en cas d'infection uro-génitale.

\section{Ocimum basilicum L. (Basilic; Lahbaq)}

Le jus de feuilles est utilisé en gouttes oculaires en cas d'irritation des yeux.

Une décoction de feuilles et de sommités fleuries est appliquée sur le cuir chevelu en cas de chute des cheveux.

Une infusion de feuilles est conseillée : constipation, ballonnement intestinal, urétrite et insomnie.

\section{Origanum compactum Benth. (Origan; Zaâter)}

La décoction est conseillée en cas de douleurs abdominales, de règles douloureuses, de ballonnement intestinal, d'inflammations rhumatismales et d'affections respiratoires.

Une préparation a base d'origan, de graines du radis (Raphanus sativus L.) et du câpre (Capparis spinosa L.), sous forme de poudre mélangée au miel, est utilisé en cas de stérilité féminine.

Origanum majorana L. (Marjolaine; Mardadouch)

Une infusion est employée en cas d'infection respiratoire, de troubles gastriques et de maux de tête.

\section{Rosmarinus officinalis L. (Romarin; Azir)}

L'infusion de romarin est utilisée en cas d'infections pulmonaires (bronchite), d'atteintes hépatiques (cirrhose, lithiase biliaire), de troubles digestifs (diarrhées) et d'infections génitales (syphilis).

En usage externe, la poudre de romarin est employée en cataplasme en cas de plaies, de brûlures et de gale.

\section{Salvia officinalis L. (Sauge; Salmiya)}

Une décoction de racines est utilisée en cas de maladies cardiaques et lors de la ménopause. 
Toxicité: La sauge officinale peut être dangereuse pour les enfants, elle peut provoquer des convulsions épileptiques (BRUNETON, 1996).

Salvia verbenaca (L.) Briq. (Fausse verveine; Khiyata)

Les feuilles en poudre sont utilisées en cataplasme pour traiter les plaies.

\section{Thymus vulgaris L. (Thym; Zâaytra)}

Une infusion de thym est utilisée pour le traitement du ballonnement intestinal, de douleurs génitales, de douleurs intestinales et en cas de diabète.

\section{Lauraceae}

Laurus nobilis L. (Laurier; Aassa moussa)

Une infusion de feuilles (une tasse après chaque repas) est utilisée en cas d'une digestion lente.

\section{Linaceae}

Linum usitatissimum L. (Lin; Zariât lkatan)

Une infusion de lin est indiquée en cas de constipation.

En cas d'asthme, des drogues, sous forme de poudre de Lin (Linum usitatissinum L.), de réglisse (Glycyrrhiza glabra L.), de graines de fenouil ( $\mathrm{Fo}$ eniculum vulgare P. Mill.) et de Nigelle (Nigella sativa L.) : $1 / 4 \mathrm{~kg}$ de chaque drogue) mélangé à un $1 / 2$ litre de miel, sont utilisées à raison d'une cuillère à café chaque matin (pour une durée de deux mois).

\section{Lythraceae}

Lawsonia inermis L. (Henné; Henna)

Les feuilles sous forme de pâte sont utilisées en application locale pour la teinture et le traitement des cheveux.

Antipelliculaires.

Cicatrisante des plaies. Le henné traite certaines dermatoses (Eczéma).

\section{Myrtaceae}

Eucalyptus globulus Labill. (Eucalyptus; Kalito, kalitous)
Une fumigation de feuilles d'Eucalyptus ( $E u$ calyptus globulus Labill.), de tiges feuillées de marrube blanc (Marrubium vulgare L.), d'ombelles de khella (Ammi visnaga Lamk.), de clous de girofle (Eugenia caryophyllata Thunb.), de feuilles de navet (Brassica rapa L.), d'oignon (Allium cepa L.) et de têtes de sardine est conseillée en cas de la fièvre typhoïde.

Une infusion de jeunes feuilles (poignet de feuilles par $1 / 2$ litre d'eau) est conseillée (une tasse le soir jusqu'à guérison) pour traiter une bronchite et des états grippaux.

Les feuilles en cataplasme (pour une durée de 10 jours) sont conseillées en cas des douleurs rhumatismales.

Eugenia caryophyllata Thunb (Girofle; Qoronfel)

Une fumigation de clous de girofle (Eugenia caryophyllata Thunb), de feuilles d'eucalyptus (Eucalyptus globulus Labill.), de tige feuillée de marrube blanc (Marrubium vulgare L.), d'ombelle de kelle (Ammi visnaga Lamk), de feuille de navet (Brassica rapa L.), d'oignon (Allium cepa L.) et de tête de poissons est efficace en cas de fièvre typhoïde.

Une infusion de clous de girofle soulage les douleurs gastriques.

La pâte de clous de girofle diminue les maux de dents.

Toxicité: le clou de girofle est déconseillé aux gens qui souffrent de l'hypertension arterille.

Myrtus communis L. (Myrte; Rihan)

Une infusion de feuilles en poudre est utilisée en cas de douleurs gastriques.

Une infusion de feuilles est utilisée en cas d'asthme.

Les feuilles de myrte réduites en poudre puis en pâte, mélangées au henné (Lawsonia inermis L.) sont appliquées sur le cuir chevelu en cas de chute de cheveux.

\section{Oleaceae}

Olea europeae L. (Olivier; Zitoun)

Une infusion de feuilles est utilisée en cas d'hypertension artérielle et de diabète. 


\section{Papaveraceae}

Papaver somniferum L. (Pavot; Kharchacha)

Une infusion de verveine (Lippia citriodora) et d'une partie de la capsule est utilisée en cas d'insomnie des nourrissons.

NB: Plante très toxique.

\section{Pinaceae}

Pinus pinaster Aiton (Pin maritime; Tayda)

En cas de brûlures, on étale une couche d'une préparation à base de 'Smen" et de poudre d'écorce.

\section{Poaceae}

Agropyrum repens $\mathrm{P}$. Beauv. (Chiendent; Njem)

Une décoction de rhizome de chiendent ajoutée à l'asperge (Asparagus officinalis L.) est utilisée en cas de stérilité féminine et de règles douloureuses.

Une décoction à base de fleurs de cactus (Opuntia ficus-indica (L.) Mill.), de stigmates de maïs (Zea mays L.), de rhizomes de chiendent (Agropyrum repens P. Beauv.) et de graines d'orge (Hordeum vulgare L.), (faire bouillir 15 à $20 \mathrm{~min}$ ) est utilisée pour traiter l'infection rénale et urinaire.

\section{Polygonaceae}

Polygonum aviculare L. (Rénouée; wedmo)

Les feuilles et les racines (sous forme de poudre) facilitent le drainage de l'abcès en cas de furonculose.

Rumex acetosa L. (Oscille; Hommida)

Une infusion de graines d'oscille en lait est utilisée en cas de diabète.

\section{Ranunculaceae}

Delphinium staphysagria L. (Staphysaigre; Habat rass)

Les graines réduites en poudre mélangées à l'huile d'olive sont appliquées sur le cuir chevelu comme traitement antichute et anti-poux.
Toxicité: Par voie interne, les graines provoquent des intoxications graves, parfois mortelles (deux cuillerées à café de poudre de graines en ingestion, provoquent le mort d'un adulte) (BELLAKHDAR, 1997).

Nigella sativa L. (Nigelle; Haba sawda, Sanouje)

La nigelle est une plante très utile en médecine traditionnelle, mais il est impératif de respecter les doses et ne pas en faire un usage prolongé vu sa toxicité. Elle est utilisée sous forme de poudre mélangée à l'huile d'olive ou au miel, toute seule ou ajoutée à d'autres drogues comme remède de différentes pathologies:

Affections respiratoires: grippe, bronchite, asthme.

Douleurs gastro-intestinales.

Kystes.

Toxicité: la toxicité de la graine à forte dose n'est pas totalement ignorée des populations, les l'utilisent souvent comme abortif (à des doses allant de 25 à 50 graines) (Bellakhdar, 1997).

Ranunculus muricatus L. (Renoncule muriquée; Ouden lhalouf)

Une décoction est utilisée en cas d'accouchement.

Toxicité: Ingérées, elles peuvent provoquer des stomatites, des brûlures, des ulcérations (BRUNETON, 1996).

\section{Rhamnaceae}

Ziziphus lotus (L.) Lam. (Jujubier; Sedra)

Les fruits frais sont consommés en cas de troubles gastriques (vomissements).

Les feuilles en infusion sont utilisées en cas de diarrhée et d'insuffisance cardiaque.

\section{Rosaceae}

Rubus ulmifolius Schott. (Ronce; aoulek)

Les fruits agissent efficacement contre les diarrhées.

Les feuilles en poudre à usage externe sont appliquées contre les brûlures. 


\section{Rubiaceae}

Rubia tinctorium L. (Garance; Fouwa)

Un décocté de racines est conseillé en cas d'anémie

La garance, le thym (Thymus vulgaris L.), le fenugrec (Trigonella foenum-graecum L.) et l'armoise blanche (Artemisia herba alba Asso.) sont utilisés sous forme de poudre en cas de diabète (petite cuillère deux fois par jour).

\section{Solanaceae}

Datura stramonium L. (Datura; Chdek ejmel)

En cas de douleurs, de névralgies et de douleurs d'utérus, les feuilles sont utilisées sous forme de cigarette.

NB: Plante très toxique.

\section{Thymelaeaceae}

Daphne gnidium L. (Garou; Lazaz)

Le garou entre dans des préparations adoucissantes des cheveux et anti-chute.

Toxicité: Les intoxications au garou sont nombreuses, surtout chez les femmes qui l'utilisent pour avorter et chez les enfants que la couleur rougeorangée des baies attire (BELLAKHDAR, 1997).

\section{Verbenaceae}

Lippia citriodora H. B. \& K. (Verveine odorante; Lwiza)

Une infusion de feuilles est conseillée aux nourrissons et aux nouveaux opérés.

Une décoction au lait est utilisée en cas de stress.

Une simple décoction est utilisée en cas de mauvaise digestion.

Verbena officinalis L. (Verveine officinale; Baymoute)

Une décoction est utilisée en cas de diarrhée et de douleurs gastriques.

\section{Zygophyllaceae}

Peganum harmala L. (Harmel; Harmel)

Une préparation à base des graines (en poudre) mélangée à l'huile d'olive est utilisée comme traitement antichute.

Une décoction est utilisée pour provoquer l'avortement.

Toxicité: Des intoxications au harmel sont signalées chez l'homme et surtout chez les enfants, par absorption des mixtures thérapeutiques traditionnelles surdosées (BELLAKHDAR, 1997). 